\title{
Model Experiments of Histochemistry of Connective Tissue, especially of Fibrinoid Change
}

\author{
Y. Mizushima \& R. Kasukawa \\ Department of Physical Therapy and Medicine, Faculty of Medicine, University \\ of Tokyo, Tokyo, JAPAN (Director : Prof. Y. Oshima)
}

\begin{abstract}
Preface: It is important to clarify what substances actually give positive reaction with Periodic Acid Schiff (PAS) and Toluidine Blue Metachromasia (TBM), which are usually employed in the recent histochemical study of connective tissue. In this series of work, chemically pure proteins and acid mucopolysaccharides, and their complexes were histochemically studied. Bovine albumin and fibrinogen, globulin from human blood, shark chondroitin sulfate, and hyaluronate were used in this experiment.
\end{abstract}

Experimentals: High molecular acid mucopolysaccharides give a strong metachromatic reaction with toluidine blue, while by hyaluronidase decomposed acid mucopolysaccharides and plasma proteins show negative result. Fibrinogen gives relatively strong reaction with PAS. Other materials do not strongly react with PAS.

Following experiment was carried out with acid mucopolysaccharide protein complexes. Both acid mucopolysaccharides and proteins were dissolved together in saline solution in concentration of $0.3 \circ$, and $\mathrm{pH}$ of the solution was adjusted below 6 by hydrochloric acid. Then acid mucopolysaccharide protein complexes were obtained in the form of precipitation, changing the ratio of protein to acid mucopolysaccharide, and also changing the grade of depolymerization of acid mucopolysaccharides. Acid mucopolysaccharide protein complexes obtained according this method gave a PAS positive reaction. When the concentration of protein increased more than 3 times of acid mucopolysaccharide, the complex gave a strong PAS reaction, whereas TBM became negative in proportion to the increase in the ratio of protein (Table 1). Complex of protein and decomposed acid mucopolysaccharide showed similar PAS reaction but negative reaction with TBM (Table 2). Complex of fibrinogen and acid mucopolysaccharide gave the strongest reaction with PAS among plasma proteins.

Table 1: PAS and TBM of acid mucopolysaccharide protein complex

\begin{tabular}{c|c|c}
\hline Albumin/Chondroitin sulfate & PAS & TBM \\
\hline 0 & - & + \\
0.75 & \pm & + \\
1.5 & + & + \\
3.0 & + & \pm \\
6.0 & + & - \\
\hline
\end{tabular}


Table 2: PAS and TBM of depolymerized acid mucopolysaccharide protein complex

\begin{tabular}{c|c|c|c|c}
\hline $\begin{array}{c}\text { Ratio of } \\
\text { intensity of } \\
\text { hyaluronidase }\end{array}$ & \multicolumn{2}{|c|}{$\begin{array}{c}\text { Depolymerized chondroitin } \\
\text { sulfate + albumin }\end{array}$} & \multicolumn{2}{c}{$\begin{array}{c}\text { Depolymerized hyaluronate } \\
\text { albumin }\end{array}$} \\
\hline 0 & PAS & TBM & PAS & TBM \\
3 & + & + & + & + \\
10 & + & + & + & + \\
20 & + & + & + & - \\
50 & + & \pm & + & - \\
\hline
\end{tabular}

Hyaluronate in the human synovial fluid combines with proteins with covalent bond. When hydrochloric acid was added to the synovial fluid, mucin clot formation by ionic bond occured. Interesting result was observed that synovial fluid did not give a strong PAS positive reaction by itself, while mucin clot gave a strong PAS reaction and negative result with TBM. (Fig. 1)

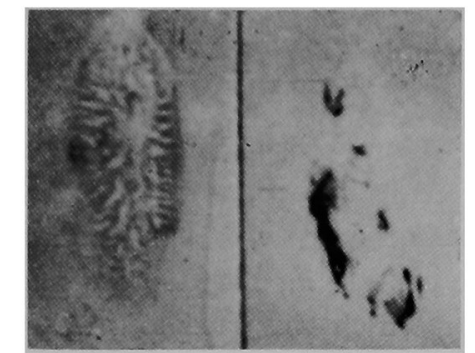

Fig. 1: PAS of synovial fluid (left) and mucin clot (right)

Discussion and Conclusion: Acid mucopolysaccharides show a positive reaction with TBM, but acid mucopolysaccharides which combine fully with proteins or low molecular acid mucopolysaccharides, even in free form, give negative result with TBM.

Chemically pure acid mucopolysaccharides do not strongly react with PAS, and Wharton's jelly of umbilical cord, which contains a large amount of hyaluronate, gives negative result with PAS reaction ${ }^{12}$. Some kind of protein actually shows positive PAS reaction, but not so strong by itself $^{23}$. In this series of work it was found that when plasma proteins were combined with acid mucopolysaccharides in acid solution, they gave a clear and strong PAS reaction. A similar interesting result was also observed in an experiment of synovial fluid and its mucin clot. It is known that $\mathrm{pH}$ of inflamed tissue becomes below 6 in some cases. This tendency might make it easier to form an ionic bondage between acid mucopolysaccharides and plasma proteins. Because a basic protein such as protamin combines with acid mucopolysaccharides even in neutral solution, but the combination of acid mucopolysaccharides with plasma proteins occurs only in acid solution. This is one of our explanation of the formation of pathological PAS positive substances, such as in fibrinoid changes. It has been postulated by Altschular ${ }^{3)}$, Ziff ${ }^{4)}$ and Pearce ${ }^{5)}$ that acid mucopolysaccharides relate to the formation of fibrinoid. 


\title{
References
}

1) Noguchi, Y. et al.: 2ed Congr. Jap. Histochem. Soc. 1961. 2) Gomori, G.: Am. J. Clin. Path., 25 : 1336, $1955 . \quad$ 3) Altschuler, C. H. et al. : Am. J. Path., 27 : 141, 1951. 4) Ziff, M. et al. : Proc. Soc. Exp. Bio. \& Med., $76: 649,1951 . \quad$ 5) Pearce, R. H. et al. : Ann. Rheumat. Dis., $15: 65,1956$.

\section{A quantitative Determination for Fluoro-intensity by Means of Fluorescent Antibody Technique}

\author{
Kitasu Suzue, Shigehisa Aoki and Michiko Suzue \\ From the First Division of Pathological Institute, Faculty \\ of Medicine, Kyoto University
}

Recently, the immunofluorescent method has been reformed to be much easy on its technique by many investigators, Riggs, Marshall et al. However, no body has described about the information of quantitative measurement on the fluoro-intensity in the cells using the fluorescent antibody technique. This paper presents an attempt which we tried to measure the fluoro-intensity in the individual cell by microspectrophotometer. Also we tried to apply this method not only to fluorescent antibody but to several histochemical studies.

\section{Principle of the method for the measurement of fluoro-intensity}

In the quantitative determination of fluoro-intensity in the cell, it is really important that experimental specimens should be arranged under the constant conditions. Namely, the thickness of the sections or specimens, the volume of fluorescent conjugate per tissue unit and other various conditions must be always identical in mutual specimens from control materials. Regulating for the thickness of sections strictly, free cells were used favourably. Moreover, when fluorescent conjugate is covered on specimens, the conjugate leans to one edge of the specimen or spread to whole over the specimens, consequently, the difference of fluorescent intensity yields. So, fluorescent reagent was used under the special cover glass which was named by us "the cover glass with the support" (Fig. 1). Thus, the specimens arranged under the several conditions

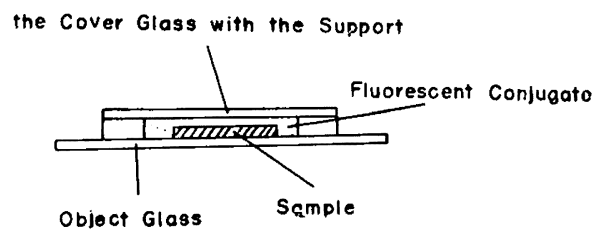

Fig. 1 shows the cover glass with the support. 